\title{
Combining Airborne and Terrestrial Laser Scanning technologies for measuring complex structures
}

\author{
Kalev Julge, Artu Ellmann \\ Tallinn University of Technology, Faculty of Civil Engineering, Dept. of Road Engineering, Chair of Geodesy, Ehitajate tee 5, Tallinn 19086, Estonia
}

\begin{abstract}
In recent years using laser scanning has gained popularity in measuring different objects, complementarily to traditional surveying and mapping methods. Terrestrial Laser Scanning (TLS) is often used for high-resolution accurate data acquisition of complex structures such as buildings, facades, etc. Airborne Laser Scanning (ALS) on the other hand can be used for remote surveying of large areas. Although TLS and ALS are generally used in separate applications, there are some possibilities where they may efficiently complement each other. In this study TLS and ALS data are combined for surveying a water tower where the façade is measured with TLS and the roof-top with standard high-elevation ALS. The advantages and shortcomings of using TLS along with ALS for measuring complex and non-standard structures are analyzed, the possible sources for errors are determined and some recommendations for methodology of field measurements and data processing in order to eliminate/reduce systematic errors are given. The estimated accuracy of combining TLS and ALS data after implementing the necessary adjustments was found to be about $5 \mathrm{~cm}$.
\end{abstract}

Keywords: LIDAR; Airborne Laser Scanning; Terrestrial Laser Scanning; Point Cloud.

\section{Introduction}

Laser scanning is a remote sensing method which utilizes Light Detection and Ranging (LIDAR). It uses laser pulses to measure distances to objects and based on these distances (and the angles of laser beams) calculates the 3D coordinates of measured points. The LIDAR device can be mounted on a tripod (terrestrial laser scanners - TLS), an aircraft (airborne laser scanning - ALS) or a ground vehicle (mobile laser scanning - MLS). In this study combining TLS and ALS is analyzed. TLS and ALS are usually used separately due to their very different characteristics, to our present knowledge there is no dedicated research published on combining them at surveying complex structures or buildings. However, a practical experiment for creating a 3D model of the medieval Old Town in Tallinn (Estonia) was accomplished in 2012. The resulting 3D model consists of over 700 buildings (and streets between them) covering an area of 121 ha. The facades of the buildings were captured with a combination of TLS and MLS at a resolution of at least 1000 points $/ \mathrm{m}^{2}$. The ALS campaign, conducted specially for the project, was helicopter-flown at a low altitude $(500 \mathrm{~m})$, thus resulting in a relatively dense point cloud of 100 points $/ \mathrm{m}^{2}[1]$.

In many countries nation-wide ALS campaigns have been launched. These are proceeded at higher elevation (up to a few kilometers), thus resulting in sparser data $\left(\sim 0.3 \ldots 1.5\right.$ points $\left./ \mathrm{m}^{2}\right)$. However, these national programmes make ALS data more accessible to a common user, as there is no need for arranging expensive single-purpose ALS campaigns. Therefore, this study analyses the possible use of standard high-altitude ALS campaigns for capturing complex structures.

Standard high-altitude ALS is generally considered not suitable for the task in hand due to the relatively low resolution and accuracy compared to TLS. Therefore it is/should be used as more of a last resort when TLS of the entire object is not feasible. TLS is much more detailed with an average spatial resolution of up to a point for every few mm, while ALS point cloud is less dense. Therefore TLS is used for surveying of in situ objects such as buildings, bridges, road surfaces, etc., whereas ALS to measure large areas such as pit mines, infrastructure objects and ground surfaces for compilation of Digital Terrain Models. In some instances some parts of the structures cannot be covered with only TLS. An example of this are high-rise buildings with pitched roofs. This means that either the scanner cannot be placed on the roof-top or the roof-top is not measurable from the ground. In these cases ALS data can be used to complete the point cloud of the structure. This case

Corresponding author: Kalev Julge. E-mail address: kalev.julge@ttu.ee

http://dx.doi.org/10.3846/enviro.2014.213

(C) 2014 The Authors. Published by VGTU Press. This is an open-access article distributed under the terms of the Creative Commons Attribution License, which permits unrestricted use, distribution, and reproduction in any medium, provided the original author and source are credited. 
study is based on laser scanning a water tower, where the facades where measured with TLS. However TLS of the roof proved to be impossible. In order to complete the point cloud, ALS data was used.

The outline of the paper is as follows. First the basic principles of laser scanning methods and aspects that affect this study are reviewed, after which some possible applications and problems of combining TLS and ALS data are discussed. Then the case study at hand is described including the methodology and achieved results. Lastly, a brief summary concludes the paper.

\section{Review on TLS and ALS principles}

TLS is used to gather high-resolution accurate spatial data about objects such as buildings, bridges, statues, road surfaces or other structures. It is especially useful in situations where the traditional surveying techniques do not provide enough accuracy or details, e.g. complicated facades, curved objects, etc. With TLS it is possible to gather data with an accuracy of $\mathrm{cm}$ or even more [2] and at a resolution up to a few $\mathrm{mm}$. However, in order to save time and decrease the file sizes, the resolution is usually set at a few $\mathrm{cm}$ depending on the complexity of the measured object.

Point clouds measured from different stations are registered, i.e. a spatial transformation that aligns different point sets by common targets or overlapping surfaces is used. The point cloud may be calculated in some known coordinate system or in some relative coordinate system specific to the certain project. In the first case, it is achieved by using coordinated geodetic reference points.

TLS can theoretically be used to capture surfaces within $300 \mathrm{~m}$ range but in practice the reasonable distance to an object is about $50-100 \mathrm{~m}$, varying according to specific scanner and characteristics of the surface. The laser beam has a tendency to diverge, i.e. the laser "foot-print" gets increasingly larger the further it travels. It is advantageous that the laser beam size remains small as it reaches a target, thus shorter ranges are preferable. This enables acquiring finer details.

In ALS the LIDAR device is placed on an aircraft, e.g. plane, helicopter or unmanned aerial vehicle (UAV). ALS is used more in applications where there is a need to quickly cover large areas, for example measuring ground surfaces for compilation of Digital Elevation Models, infrastructure objects, etc., or areas otherwise inaccessible, such as deserts or glaciers. As a rule, ALS point cloud is much less dense than a TLS point cloud and varies usually from $0.1 \ldots 20$ points per $\mathrm{m} 2$ depending on many factors. Basically, acquiring more high-resolution data requires more time, flight hours and most importantly more funds.

In order to determine the position and orientation of the aircraft at any moment, a Global Navigation Satellite System (GNSS) receiver and an Inertial Measurement Unit (IMU) are used. GNSS receiver measures the position and IMU records the pitch, roll and yaw of the aircraft. Since the coordinates from GNSS are used then the resulting point cloud is in geodetic coordinates and can be transformed to any local system. Given that the calculation involves so many parameters, then the accuracy of ALS is less than that of TLS, usually estimated at $5-15 \mathrm{~cm}$ in favorable conditions [3][4].

As ALS is generally performed at an altitude of $1000 \ldots 4000 \mathrm{~m}$, the divergence of the laser beam is much more pronounced and can reach up to $0.5 \mathrm{~m}$ in diameter. This means that capturing fine detail is impossible and it can cause some measurements errors on surfaces that are at an angle to the laser beam as it hits a large area that is not the same distance from the LIDAR device.

In many countries nation-wide ALS campaigns have been launched. Some are an on-going process, some have been completed and some are already on next cycles. Examples in Europe include Germany, Netherlands, Austria [5], Switzerland [6], Finland [7] and Estonia. ALS is cost effective when used to cover very large areas, so for using ALS in small projects is usually not practical. This existing data makes ALS much more accessible. Using UAV as a platform for laser scanning has evolved recently and with further refinement can probably become a viable cost-effective option for use in smaller applications.

\section{Combining TLS with ALS - advantages, shortcomings and applications}

In some cases combining these laser scanning technologies can be useful - in applications where it is not possible to cover the entire object with only one method or in projects where for different steps of the process, different methods are better suited. For example, in creating 3D models of buildings, structures or built environment as a whole. With TLS it is possible to gather high-resolution data about building facades but the roofs are most likely not visible and often not accessible. Contrastingly in ALS data, the roof-tops are captured well, whereas there is little usable data about the facades.

Another use would be monitoring the volume of earthworks at large sites. ALS could be used to create the initial model of the whole area, where as TLS could be used to monitor the cut and fill volumes during the process. It would also be possible to monitor coastal processes by using ALS to detect whether there are significant changes and in case there are, to use TLS to accurately measure the extent of the changes. Another example would be combining ALS and TLS to monitor the extent of land slides or debris flows by using ALS data to reconstruct a pre-event surface and TLS to measure and create a post-event surface [8]. These are just a few possible examples.

Now although combining TLS with ALS obviously has potential, there are some issues. ALS data is much less dense and less accurate than TLS. In applications where the desired accuracy and data resolution are that of TLS, ALS does not meet the expected demands. Also given the relatively large differences in spatial resolution, the methods for data processing 
have to be altered to best suit the data in hand. This is especially pronounced while using automatic filtering, segmenting, modelling, etc. algorithms that require the use different parameters depending on the point cloud density.

Additionally, combining different laser scanning methods raises a few accuracy problems. The main issue is how to ensure that the TLS and ALS data are aligned to each other, i.e. there is no systematic errors present between them. First of all, TLS could adopt an arbitrary coordinate system where the $\mathrm{x}, \mathrm{y}, \mathrm{z}$ coordinates as well as the orientation are random. This saves time from coordinating reference points and is usually proceeded if the location of the object in the national coordinate system is not required. ALS results are most commonly always referred to national geodetic reference frame.

Even if the TLS and ALS point clouds are using the same coordinate system then there is still a possibility that systematic errors would occur. This is caused by the fact that most likely TLS and ALS measurements are done by using different reference marks. For example the TLS is done by using geodetic points on the ground and ALS uses data from GNSS systems and IMU to calculate the position and orientation.

The problem of unifying ALS and TLS point clouds could be easily solved by finding distinguishable points in both point clouds and moving one of the clouds to the correct location. Unfortunately, ALS data is usually not dense enough to accurately determine the coordinates of a certain point. Therefore it is necessary to find the value of the systematic errors by other means.

The systematic error of elevation can be determined by finding horizontal surfaces (e.g. a nearby parking lot) that are present on both point clouds. By comparing the elevation differences between the same surface measured by TLS and ALS, the extent of the systematic error should become evident. Points near the edge of the surface should be discarded to minimize the errors caused by shifts at the $\mathrm{x}$, $\mathrm{y}$ plane and the large size of the ALS laser beam footprint on the ground caused by divergence. For example, a part of the ALS pulse might reflect from a curbstone instead of the pavement. The larger the surface area the better because a larger sample amount reduces the influence of inaccurate points.

Eliminating systematic errors in planar coordinates can be a bit more complicated. As mentioned earlier, ALS point clouds tend not to be dense enough to accurately determine certain points or edges. For this the ALS data need to be processed first. The aim is to find planar surfaces that meet, e.g. facets of a pitched roof. Now although the edge is not clearly defined in the point cloud (Fig. 1a), the points on the surfaces can be used to construct planes and the line of intersection between these planes is the edge (Fig. 1b). If there are three intersecting planes then this results in a single corner point.

Using these common edges or points, it is possible to match the location in ALS and TLS data and register the point clouds. Obviously, this method can be proceeded only in urban areas where there are clearly defined artificial sharp edges. Finding clearly defined natural features in non-built areas is unlikely though. Also since these edges and points are most likely found on roofs in ALS data, then there is no guarantee that they are visible in TLS point clouds.

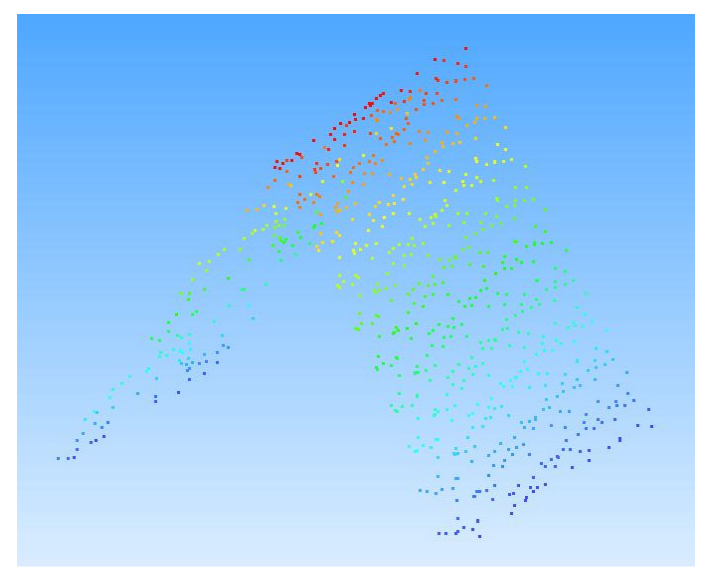

(a)

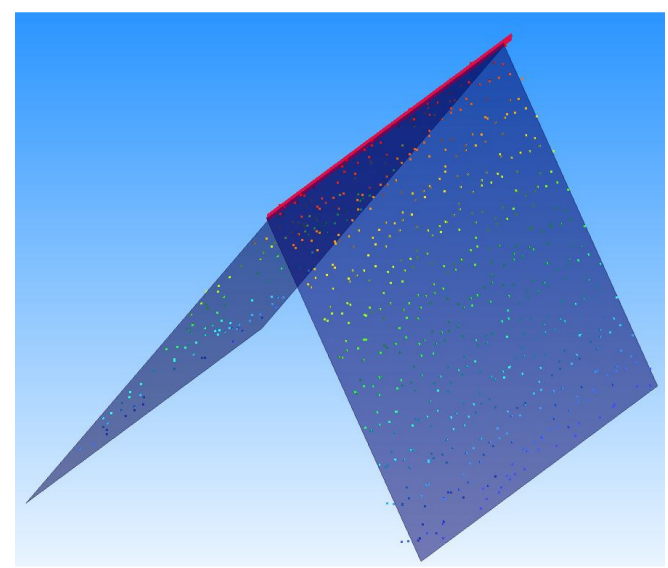

(b)

Fig. 1. Finding edges from (a) an ALS point cloud of a pitched roof by (b) constructing planes on roof facets and finding the intersection (red line)

\section{Case study}

This study tackles a TLS survey of a $30 \mathrm{~m}$ tall historic water tower (Fig. 2a). In this case the scanning of the roof-top proved to be impossible because the cone shaped pitched roof of the high-rise water tower was not visible from the ground and it was impossible to place the scanner tripod on it. Note also, that the surrounding buildings were lower than the water tower, thus it would not have been beneficial to place the scanner on a balcony or a roof of a neighboring building. So the only possibility was using ALS data to complete the point cloud of the water tower (Fig. 2b).

The TLS facade survey was conducted with a pulse-based Leica ScanStation C10. The resolution of the TLS data was 2500 points $/ \mathrm{m}^{2}(2 \times 2 \mathrm{~cm})$. The ALS data were received from the Estonian Land Board which uses a Leica ALS50-II airborne laser scanner. The ALS data tested originated from four different years - 2008, 2009, 2012 and 2013 (see also Table 1). The 2008 campaign was flown at a height of $1500 \mathrm{~m}$ and the point density was 1.2 points $/ \mathrm{m}^{2}$. The other three campaigns had a flight height of $2400 \mathrm{~m}$ with resulting point density of $0.3 \ldots 0.4$ points $/ \mathrm{m}^{2}$. 


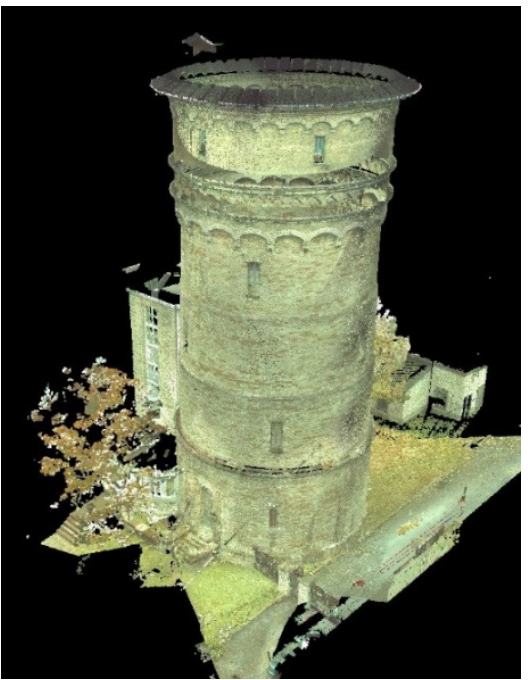

(a)

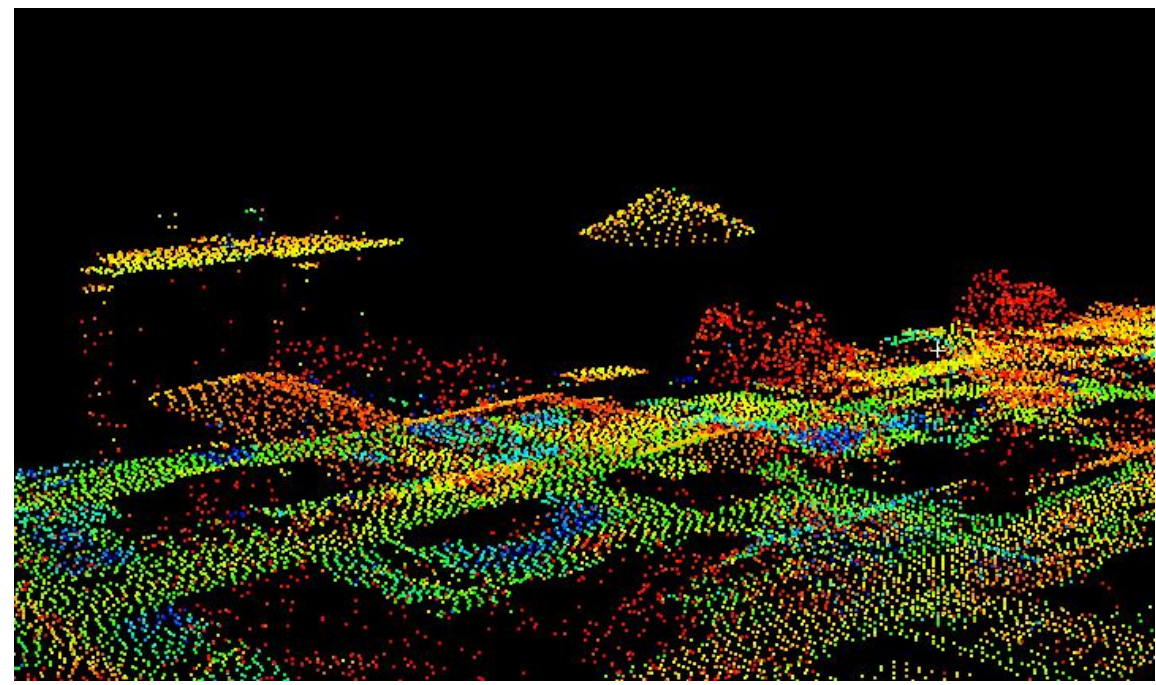

(b)

Fig. 2. (a) Point cloud of TLS data where the roof-top is missing. (b) Point cloud of ALS data where the cone shaped roof is clearly visible

In order to take into account the possible systematic errors in elevation, three horizontal reference surfaces near the water tower were selected (Fig. 3a). One of these was a $200 \mathrm{~m}^{2}$ parking lot right next to the water tower (surface 1) and the others were terraces of a large building with an area of $100 \mathrm{~m}^{2}$ each (surface $2 \& 3$ ) located some $125 \mathrm{~m}$ away. These were the only horizontal surfaces near-by that had remained unchanged (no roadwork, etc.) since the first ALS flight in 2008. The reference surfaces were measured with a regular $1.5 \mathrm{~m}$ steps by a total station using the same initial points that were used for the TLS orientation.

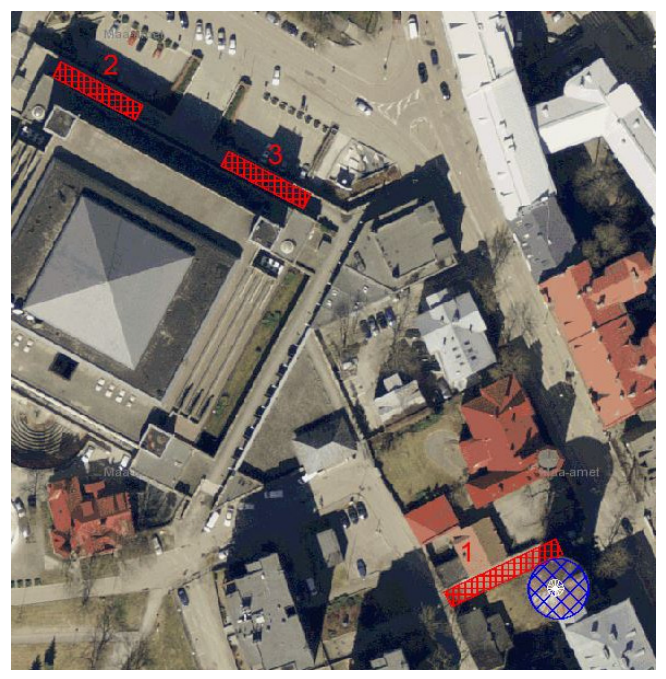

(a)

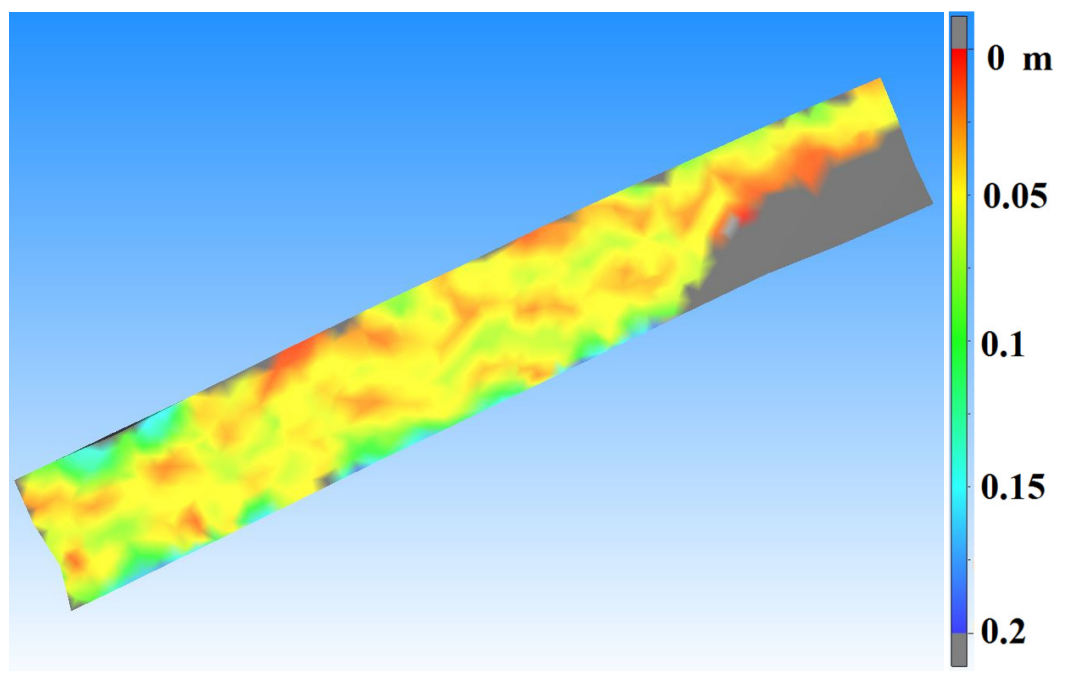

(b)

Fig. 3. (a) The location of the ALS reference surfaces (red hatch) with respect to the water tower (blue hatch). (b) Elevation differences of 2008 ALS points from the reference surface 1 . ALS points are on average $0.05 \mathrm{~m}$ higher than the reference surface

\section{Data processing and results}

In order to find the systematic errors in elevation, the three surfaces were separated from the rest of the ALS point cloud and obvious measurement blunders were removed. Based on these cleaned point clouds Triangulated Irregular Network (TIN) models were formed. TIN models derived from ALS data were compared to reference TIN models derived from total station measurements in TLS coordinate system. The average elevation differences were found along with the standard deviation, cf. Table 1 and Fig. $3 b$.

It was found that the detected discrepancies between the less dense $\left(0.3 \ldots 0.4\right.$ points $\left./ \mathrm{m}^{2}\right)$ ALS data (the 2009,2012 and 2013 flights) and reference surfaces appeared to be inadmissible. The standard deviations of detected discrepancies were also considerably large. The average of discrepancies exceeded $10 \mathrm{~cm}$ and standard deviations $4 \mathrm{~cm}$, thus it was impossible to accurately estimate the presence and values of systematic errors and shifts in elevation. To use the sparser data, larger reference surfaces are required to increase the sample size, which would allow reducing the influence of measurement errors. This would enable more accurately determining the systematic error values. 
Table 1. The statistics of ALS data and the comparisons between the ALS and reference surfaces

\begin{tabular}{llllll}
\hline \multicolumn{2}{l}{ ALS data } & \multicolumn{4}{l}{$\begin{array}{l}\text { Average elevation difference between the ALS and reference surfaces [m] / No. of ALS points on reference } \\
\text { surface / Standard deviation }[\mathrm{m}]\end{array}$} \\
\hline Year & $\begin{array}{l}\text { Flight height } \\
{[\mathrm{m}]}\end{array}$ & $\begin{array}{l}\text { No. of ALS } \\
\text { roof-top points }\end{array}$ & $\begin{array}{l}\text { Reference surface } 1 \\
\left(200 \mathrm{~m}^{2}, 51 \text { ground survey }\right. \\
\text { points })\end{array}$ & $\begin{array}{l}\text { Reference surface 2 } \\
\left(100 \mathrm{~m}^{2}, 36 \text { ground survey }\right. \\
\text { points })\end{array}$ & $\begin{array}{l}\text { Reference surface } 3 \\
\left(100 \mathrm{~m}^{2}, 38 \text { around survey }\right. \\
\text { points })\end{array}$ \\
\hline 2008 & 1500 & 225 & $0.054 / 181 / 0.028$ & $0.051 / 94 / 0.024$ & $0.047 / 107 / 0.018$ \\
2009 & 2400 & 62 & $0.111 / 54 / 0.059$ & $0.040 / 27 / 0.027$ & $0.060 / 25 / 0.036$ \\
2012 & 2400 & 61 & $0.015 / 54 / 0.041$ & $-0.016 / 24 / 0.033$ & $0.003 / 25 / 0.030$ \\
2013 & 2400 & 50 & $0.028 / 55 / 0.045$ & $0.011 / 25 / 0.038$ & $0.015 / 26 / 0.035$ \\
\hline
\end{tabular}

Using the distorted data would have probably decreased the accuracy by adding more noise to the point cloud. Since these ALS campaigns were flown at a height of $2400 \mathrm{~m}$ as opposed $1500 \mathrm{~m}$ of the 2008 campaign, the laser beam divergence was larger resulting in less accurate measurements. Also there were fewer points which magnifies the influence of erratic ALS points. This makes it difficult to correctly register TLS and ALS point clouds which would in return distort the model of the object (Fig. 4).

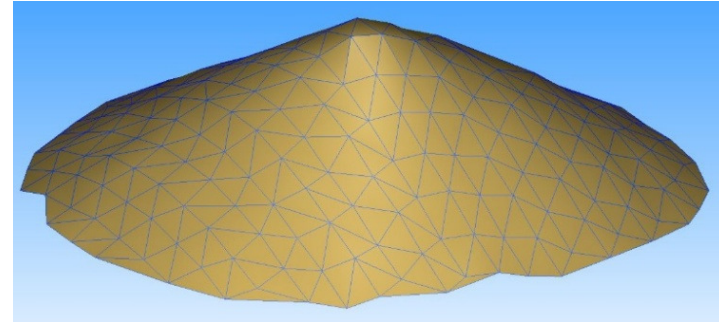

(a)

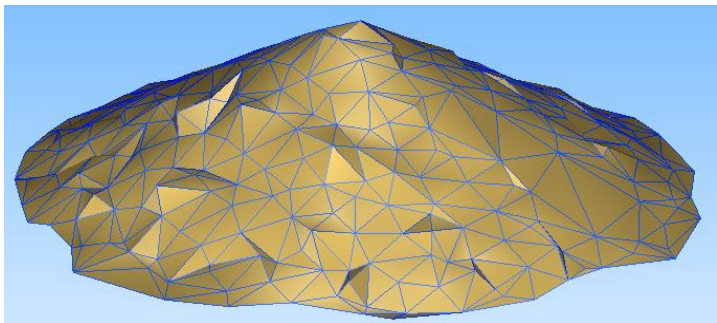

(b)

Fig. 4. Mesh models of the roof-top (a) based on the ALS 2008 points only, (b) based on all ALS points from the four campaigns illustrating the distortions to the model shape caused by alignment and measurement errors

Since it was impossible to distinguish the values of the systematic errors, the measurements seemed to be less accurate and there was a risk of incorrect alignment, then it was decided to discard the 2009, 2012 and 2013 ALS data and use only denser 2008 ALS data for modeling the shape of the roof-top.

In more dense (1.2 points $\left./ \mathrm{m}^{2}\right)$ ALS data from 2008, the elevation differences were more uniform and a systematic bias as of $0.051 \mathrm{~m}$ was detected (Fig. 3b). In other words, the ALS data appeared to be higher than the TLS data. This 1D bias was removed from the ALS point cloud to conform with TLS data. The resulting average standard deviation between the two datasets obtained was $2.3 \mathrm{~cm}$, cf. Table 1 .

Based on this, it can be presumed that a point density of at least 1 point $/ \mathrm{m}^{2}$ is required to properly register different point clouds. If this is the case, then finding a systematic error is possible. Otherwise the data is too sparse and inaccurate to distinguish a clear trend with any certainty.

This left the problem of the alignment in the horizontal $\mathrm{x}, \mathrm{y}$ plane. The roof-top was not visible from the ground and therefore was not represented in TLS point cloud either. Unfortunately, no other features (point, edge) were clearly distinguishable in ALS point cloud either, thus finding common points in TLS and ALS data was impossible. However, since the water tower was circular with a cone shaped roof, then this allowed to align the centers of the tower (originating from the TLS data) and that of the roof (ALS data).

The planar coordinates of the tower's center were found by calculating the center point of a circle that represented the outline of the tower's cross-section. The roof's center was not distinguishable directly from the point cloud or the TIN model of the roof (see also Fig. 4a). Instead the center was determined by modelling a best fit cone based on the roof-top points. In this case the cone's vertex defines also the center of the roof.

The resulting best fit cone is still an approximation. To find the extent of deviations, the root mean square error (RMSE) of the modeled cone with respect to the ALS points was found using:

$$
R M S E=\sqrt{\frac{\sum_{1}^{n} \theta_{i}^{2}}{n}}
$$

where $\theta i$ is the distance of $i$-th ALS point from the best fit cone along the surface normal, $n$ is the number of observations (225 ALS points in this particular case, see Table 1) and $i=1,2, \ldots, n$.

The RMSE was found to be $0.050 \mathrm{~m}$ with the errors distributed quite evenly between $-0.1 \ldots 0.1 \mathrm{~m}$ (Fig. 5). The accuracy of approximating a shape depends on the relative accuracy of point coordinates, as well as the number of available points. 
The more accurate and high-resolution the data are, the better results of the best fit shape approximation can be achieved. This means that modelling based on sparse ALS point clouds can cause significant errors. First, because there are larger gaps between points, yielding thus that some details get lost. Secondly, a smaller amount of points reduces the quality of shape approximation.

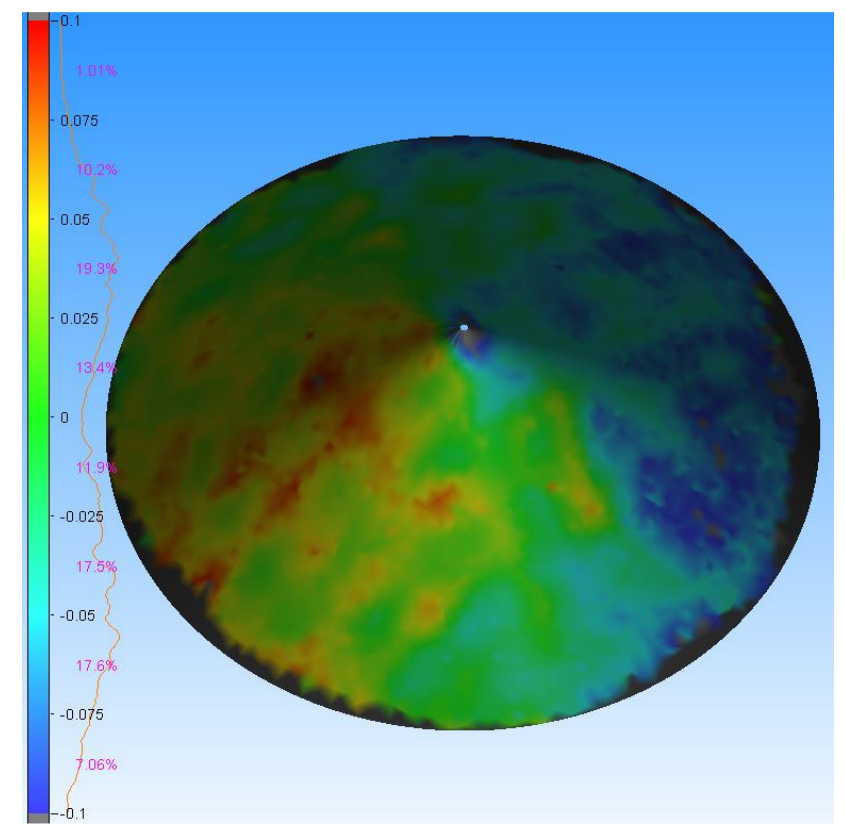

Fig. 5. Comparison between the best fit cone model of the roof-top and the initial TIN model derived from the 2008 ALS points. The root mean square error of discrepancies RMSE $=0.05 \mathrm{~m}$. Next to the legend is a line histogram presenting the percentages of deviations from the cone in the given range

Approximating shapes can potentially cause additional shifts and errors. This was also evident in this case, since the best fit cone had to be shifted horizontally by as much as $25 \mathrm{~cm}$ with respect to the center of the tower (Fig. 6a). Such a large shift is unlikely to be only caused by measurement errors or systematic errors between TLS and ALS data. The shift in planar coordinates of ALS data was adjusted by moving the best fit cone in the 2D horizontal plane, so that the cone vertex coincided with the tower's center. This method can potentially be performed with other types of roofs as well. For example, aligning the ridge of a gabled roof with the center line of a building.

By taking into consideration the systematic errors and shifts, it is possible to unify the TLS and ALS point clouds (Fig. 6b). Although it is clear that ALS data is not as high-resolution nor accurate as TLS data, ALS can still be useful if there are no other means to measure certain objects. It is usually not possible to capture fine details with standard ALS campaigns, as the point density is not sufficient to clearly distinguish small objects, e.g. chimneys, drainage elements, ventilation equipment, etc. For example, in this case, the gable dormer on the roof is almost indistinguishable in the ALS data.

Therefore ALS is useful in modelling rough shapes of roofs, when the shape can be approximated by using different shapes, e.g. planes, cones, pyramids, etc. Considering all the possible errors - measurement errors; systematic errors in elevation and in planar coordinates between TLS and ALS; shape approximation errors and shifts caused by shape approximation, then the accuracy of combining TLS and ALS data can reach ca $5 \mathrm{~cm}$. This is after making the necessary adjustments to correct (or at least reduce) these errors and it also depends on the quality of data. Further research with a larger sample size is necessary to confirm this estimation. If there are no distinguishable features (common points, edges, horizontal surfaces) to register different point clouds and reduce systematic errors, then the accuracy reduces significantly.

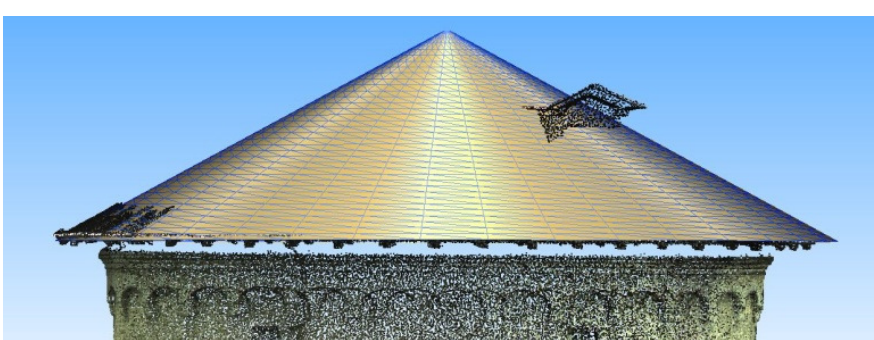

(a)

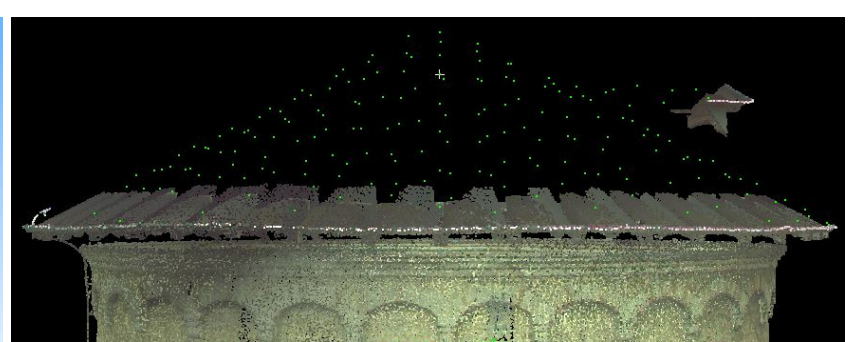

(b)

Fig. 6. (a) Best fit cone model of the roof-top before eliminating the shift. Note the TLS points of roof soffit are showing through the roof surface on the left-hand side. (b) Combined point cloud of the water tower, both TLS and ALS data are used.

Note the different resolutions of ALS and TLS data. The gable dormer partially captured with TLS is almost indistinguishable in ALS data 


\section{Conclusions}

TLS and ALS are usually used in different applications due to the difference in their characteristics. However, there are some cases where their combination could be beneficial. In this study ALS data made possible the completion of the point cloud of a water tower.

ALS data becomes much more accessible for practical use, provided that there are ALS data from country-wide campaigns available. Otherwise the expenses of single-purpose ALS campaigns might be too large to be practical and feasible. In standard ALS campaigns capturing as fine details is usually impossible. This is caused by the lower resolution of the data and the larger divergence of the laser beam. Also based on this study, significantly better results can be achieved if the point cloud density is at least 1 point $/ \mathrm{m}^{2}$. Even though fine detail is lost, the basic shapes can be approximated by using best fit shapes - planes, cones, pyramids, etc.

In order to find the shifts and systematic errors between TLS and ALS data, common features need to be distinguished from both point clouds. Finding common points outright from the point clouds is usually impossible because the resolution of the data is not sufficient. Therefore other methods are to be used. To find the extent of elevation systematic errors, horizontal surfaces that are present in both point clouds can be used by comparing the average elevations between the surfaces. A clear trend should be looked for. If the discrepancies are very large then that suggests measurement errors and the results might be distorted. To find the shifts in planar coordinates, common edges should be identified. This can be achieved by constructing intersecting planes using surfaces in the point cloud (or some other approximate shape and surface). The intersection line between planes is the edge. By finding a number of these, there is a possibility to register the point clouds. In this study the planar shift was corrected by approximating a cone and aligning it with the center of the tower.

The estimated accuracy of combing TLS and ALS data, after adjusting for the systematic errors, is about $5 \mathrm{~cm}$, depending also on the quality of data. If there is no possibility to find the values of systematic errors, then the accuracy reduces significantly. TLS data are significantly higher-resolution and more accurate than ALS data, thus with TLS most of the details are captured, whereas with ALS fine detail is normally lost. Therefore modelling based on TLS and ALS data is slightly different caused by the different resolutions. Modelling based on ALS requires more approximation and interpolating, whereas modelling based on TLS is more precise. This will be further looked at in upcoming research.

\section{Acknowledgements}

This study is supported by the Estonian Environmental Technology R\&D Programme KESTA, research project ERMAS AR12052. The ALS data used in this study was received from the Estonian Land Board under the license contract ST-A12422, 22/06/2012. The used Leica ScanStation C10 and licensed data processing software were acquired within frames of the Estonian Research Infrastructures Roadmap object Estonian Environmental Observatory (funding source 3.2.0304.110395, project No. AR12019). The prime author is supported by a NORDPLUS co-operation project AGES (Access to Geodetic Education for Society, HI-2012_1A-30040).

\section{References}

[1] Terratec Terrapro. 2012. Report of Aerial Photography, Airborna Laser Scanning, Mobile Laser Scanning, Terrestrial Laser Scanning for City of Tallinn.

[2] Dorninger, P.; Szekely, B.; Zamolyi, A.; Roncat, A. 2011. Automated Detection and Interpretation of Geomorphic Features in LiDAR Point Clouds, Vermessung \& Geoinformation 2011(2): 60-69.

[3] Gruno, A.; Liibusk, A.; Ellmann, A.; Oja, T.; Vain, A.; Jürgenson, H. 2013. Determining sea surface heights using small footprint airborne laser scanning, in Remote Sensing of the Ocean, Sea Ice, Coastal Waters, and Large Water Regions 2013 (Conference 8888), Dresden, Germany, 2013. Spie - International Society For Optical Engineering. http://dx.doi.org/10.1117/12.2029189

[4] Huising, E. J.; Gomes Pereira, L. M. 2004. Errors and accuracy estimates of laser data acquired by various laser scanning systems for topographic applications, ISPRS Journal of Photogrammetry and Remote Sensing 53(5): 245-261. http://dx.doi.org/10.1016/S0924-2716(98)00013-6

[5] Mandlburger, G.; Vetter, M.; Milenkovic, M.; Pfeifer, N. 2011. Derivation of a countrywide river network based on Airborne Laser Scanning DEMs results of a pilot study, in Proc. of the 19th International Congress on Modelling and Simulation, Perth, Australia, $2011,2423-2429$.

[6] Artuso, R.; Bovet, S.; Streilein, A. 2003. Practical Methods for the Verification of Countrywide Terrain and Surface Models, in The internal archives of photogrammetry, remote sensing and spatial information sciences, Dresden, Germany, 2003.

[7] Ahokas, E.; Kaartinen, H.; Hyyppä, J. 2008. On The Quality Checking of the Airborne Laser Scanning Based Nation Wide Elevation Model in Finland, in The internal archives of the photogrammetry, remote sensing and spatial information sciences, Beijing, China, 2008, 267-270.

[8] Bremer, M.; Sass, O. 2012. Combining airborne and terrestrial laser scanning for quantifying erosion and deposition by a debris flow event, Geomorphology 138: 49-60. http://dx.doi.org/10.1016/j.geomorph.2011.08.024 